\title{
ON THE ROBUST OPTIMAL DESIGN AND CONVERGENCE SPEED ANALYSIS OF ITERATIVE LEARNING CONTROL APPROACHES
}

\author{
Jian-xin $\mathrm{Xu}^{*}$ and Ying Tan \\ * Department of Electrical and Computer Engineering, \\ National University of Singapore, \\ Email: elexujx@nus.edu.sg
}

\begin{abstract}
In Iterative Learning Control design, convergence speed along the iteration domain is one of the most important performance factors. In this paper, we aim at achieving fastest convergence speed (time-optimal) for a variety of nonlinear nonaffine Single-Input-Single-Output (SISO) plants, and focus on the family of the linear type iterative learning controllers, including first-order ILC and higher-order ILC. The control objective can be formulated as a kind of robust optimization: optimizing the worst case performance in the presence of the interval uncertainties. To quantify convergence speed, a learning performance index - $Q$-factor - is employed. The optimal learning gain is then obtained by solving a Min-max problem. From the robust optimal design, we also reach the following conclusion: under the same interval uncertainty and applying the same min-max design which is robust and optimal, the $Q$-factor of ILC sequences of lower order ILC is always less than that of higher order ILC in terms of time-weighted norm. In the sequel, the first order ILC achieves the fastest convergence speed in the iteration domain.
\end{abstract}

Keywords: Convergence speed analysis, $Q$-factor, robust optimization

\section{INTRODUCTION}

In near two decades ILC has been extensively studied, achieves significant progress in both theory and application, and becomes one of the most active fields in intelligent control and system control (Arimoto, 1985; Bien and Huh, 1989; Kuc et al., 1992; Longman and Lo, 1997; Moore, 1998; Xu, 1997). The major objective in learning control is to design a sequence of control inputs, which ensure convergence of the tracking error along the iteration domain, in the sequel improve the time domain transient response. In this paper, we pay attention to ILC convergence speed, an important performance index in the learning control design. The fastest convergence speed is always prefered. Thus the control objective is not only to ensure the convergence of the tracking error, but to optimize the convergence speed along the iteration domain. To quantify the convergence speed, a rigorous mathematical definition of convergence speed for iterative processes $-Q$-factor, is employed in the form of objective function. Because of the existence of system uncertainties, ILC design now becomes a kind of robust optimization (Minmax), which optimizes the convergence speed while considering the worst case system uncertainties. To limit our discussion, a class of the nonlinear non-affine SISO dynamic systems with interval uncertainties will be considered. We will find a optimal learning gain achieving the fastest convergence speed for a family of linear-type ILC scheme including both first-order ILC scheme and higher-order ILC schemes. The analysis results shows that under the same interval uncertainty and applying the same Min - max design which is robust and optimal, the $Q$-factor of ILC sequences of lower order ILC is always less than that of higher order ILC in terms of the time-weighted norm. In the sequel, the first order ILC achieves the fastest convergence speed in the iteration domain. 
This paper is organized as follows. Section 2 introduces some basic knowledge and concepts to be used in subsequent sections. The learning control problem is formulated in Section 3. Section 4 presents the optimal learning gain design for linear-type ILC scheme. Illustrative examples are provided in Section 5.

\section{PRELIMINARY}

In this section, the convergence speed definition for the iteration sequences is first given, which defines the objective function in learning control design. The important Propositions in the convergence analysis of linear-type ILC scheme are also provided.

Definition 1. Let $\mathbf{x}_{k} \subset R^{n}$ be any convergent sequence with limit $\mathbf{x}^{\star}$. Then the quantities

$Q_{p}\left\{\mathbf{x}_{k}\right\}= \begin{cases}0 & \text { if } \mathbf{x}_{k}=\mathbf{x}^{\star}, \text { f.a.b.f.m. } k \\ \lim _{\substack{k \rightarrow \infty \\+\infty}} \sup \frac{\left\|\Delta \mathbf{x}_{k+1}\right\|}{\left\|\Delta \mathbf{x}_{k}\right\|^{p}} & \text { if } \mathbf{x}_{k} \neq \mathbf{x}^{\star}, \text { f.a.b.f.m. } k \\ \text { otherwise, }\end{cases}$

defined for all $p \in[0, \infty)$, are the quotient convergence factors, or $Q$-factors with respect to the norm $\|\cdot\|$ on $R^{n}$. Here $\Delta \mathbf{x}_{k} \triangleq \mathbf{x}_{k}-\mathbf{x}^{*}$ and f.a.b.f.n.m.k stands for for all but finitely many $k$.

Definition 2. Let $\mathcal{I}_{1}$ and $\mathcal{I}_{2}$ be iterative processes with the same limit point $\mathbf{x}^{\star}$, and let $Q_{p}\left(\mathcal{I}_{1}, \mathbf{x}^{\star}\right)$ and $Q_{p}\left(\mathcal{I}_{2}, \mathbf{x}^{\star}\right)$ be the corresponding Q-factors computed in the same norm on $R^{n}$. $\mathcal{I}_{1}$ is $Q$-faster than $\mathcal{I}_{2}$ if there is a $p \in[1, \infty)$ such that $Q_{p}\left(\mathcal{I}_{1}, \mathbf{x}^{\star}\right)<Q_{p}\left(\mathcal{I}_{2}, \mathbf{x}^{\star}\right)$.

Proposition 1. (Chen et al., 1998) Suppose a real positive series $\left\{c_{n}\right\}, n=1, \cdots$ satisfies

$$
\begin{array}{r}
c_{n} \leq \beta_{1} c_{n-1}+\beta_{2} c_{n-2}+\cdots+\beta_{N} c_{n-N}, \quad \forall \beta_{j}>0 \\
j=1, \cdots, N,
\end{array}
$$

where $\sum_{j=1}^{N} \beta_{j}<1$, then $\lim _{n \rightarrow \infty} c_{n}=0$.

Proposition 2. When $q=\frac{2 p}{\alpha_{2}+\alpha_{1}}, J=\min _{q \in R} \max _{g_{u} \in \mathcal{D}} \mid p-$ $q g_{u} \mid$ reaches its minimum

$$
J=|p| \theta
$$

where $\theta \triangleq \frac{\alpha_{2}-\alpha_{1}}{\alpha_{2}+\alpha_{1}}, 0<\theta<1, \mathcal{D}=\left[\alpha_{1}, \alpha_{2}\right], 0<$ $\alpha_{1} \leq \alpha_{2}$

Proof: The proof of Proposition 2 is given in Appendix A.

\section{PROBLEM FORMULATION}

Consider the nonlinear dynamic system,

$$
\begin{aligned}
\dot{\mathbf{x}}(t) & =\mathbf{f}(\mathbf{x}(t), u(t), t) \quad \mathbf{x}_{i}(0)=\mathbf{x}_{0} \\
y(t) & =g(\mathbf{x}(t), u(t), t),
\end{aligned}
$$

where $t \in[0, T], \mathbf{x}(t) \in R^{n}, y(t) \in \mathcal{R}, u(t) \in \mathcal{R}, \mathbf{f}(\cdot)$ and $g(\cdot)$ are partially unknown functions. The system is satisfying the following assumptions.

Assumption 1: Denoting $\Omega_{1} \triangleq R^{n} \times R \times[0, T]$, $0<\alpha_{1} \leq \frac{\partial g}{\partial u} \leq \alpha_{2}$ and $\left\|\frac{\partial g}{\partial \mathbf{x}}\right\| \leq M_{\mathbf{x}}, \forall(\mathbf{x}, u, t) \in \Omega_{1}$. Here $\alpha_{1}, \alpha_{2}$ are known constants and $M_{\mathbf{x}}$ is a known constant.

Remark 1. $\frac{\partial g}{\partial u}$ is equivalent to system direct feedthrough term and represents the system gain. $0<$ $\alpha_{1} \leq \frac{\partial g}{\partial u}$ warrants no singularity in the system control. $\frac{\partial g}{\partial u} \in \mathcal{D}=\left[\alpha_{1}, \alpha_{2}\right]$ indicates the presence of an interval uncertainty in the system gain, which directly affects the control performance. One of the objectives of this paper is to present an appropriate learning control design so as to achieve both robustness and optimality.

Assumption 2: Nonlinear vector function $\mathbf{f}(\mathbf{x}, u, t)$ is global Lipschitz continuous with respect to $\mathbf{x}$ and $u$ in the set $\Omega_{1}$, i.e.,

$\left\|\mathbf{f}\left(\mathbf{x}_{1}, u_{1}, t\right)-\mathbf{f}\left(\mathbf{x}_{2}, u_{2}, t\right)\right\| \leq f_{0}\left[\left\|\mathbf{x}_{1}-\mathbf{x}_{2}\right\|+\left|u_{1}-u_{2}\right|\right]$

where $f_{0}$ is an unknown Lipschitz constant.

Assumption 3: The initial resetting condition holds for all iterations, i.e., $y_{i}(0)=y_{d}(0), \forall i \in \mathcal{N}$. Here $i$ denotes the iteration number, $i=1,2, \cdots \mathcal{N}$ denotes the set of positive integers.

Assumption 4 For the given trajectory $y_{d}(t)$, there exists a unique $u_{d}(t)$ such that

$$
\begin{aligned}
\dot{\mathbf{x}}_{d}(t) & =\mathbf{f}\left(\mathbf{x}_{d}(t), u_{d}(t), t\right) \\
y_{d}(t) & =g\left(\mathbf{x}_{d}(t), u_{d}(t), t\right),
\end{aligned} \quad \forall t \in[0, T],
$$

The dynamics of the nonlinear system (2) is repeatable over a finite time interval $[0, T]$. The family of lineartype ILC scheme is constructed as

$$
u_{i+1}(t)=\sum_{k=1}^{m} p_{k} u_{i-k+1}+\sum_{k=1}^{m} q_{k} \Delta y_{i-k+1}
$$

where $m$ is a finite constant, representing the order of linear-type ILC scheme, $p_{k}$ and $q_{k}, \forall k=1, \cdots, m$ are learning gains. The objective in this paper is to find optimal learning gains $p_{k}^{*}$ and $q_{k}^{*}$ such that the convergence speed is the fastest, i.e. the $Q$-factor is the smallest. 


\section{OPTIMAL LEARNING GAIN DESIGN FOR LINEAR-TYPE ILC SCHEME}

For dynamic system (2) with the linear-type ILC scheme (4), it is well-known that if the following conditions are satisfied,

$$
\begin{aligned}
& \sum_{k=1}^{m} p_{k}=1 \\
& \sum_{k=1}^{m}\left|p_{k}-q_{k} g_{u}\right|<1,
\end{aligned}
$$

the ILC law (4) is convergent in the sense of timeweighted norm.

By ignoring the dynamic impact, i.e., those $\Delta \mathbf{x}_{i}$ terms, we have

$$
\begin{aligned}
\left|\Delta u_{i+1}\right|_{\lambda} & =\max _{t \in\left[0, T_{f}\right]} e^{-\lambda t}\left|u_{i+1}(t)-u_{d}(t)\right| \\
& \leq \sum_{k=1}^{m}\left|p_{k}-q_{k} g_{u}\right|\left|\Delta u_{i-k+1}\right|_{\lambda}
\end{aligned}
$$

The control input sequence $u_{i}(t)$ will converge to the desired $u_{d}(t)$, as a sequence, $y_{i}(t) \rightarrow y_{d}(t)$ (Bien and Huh, 1989). There may exist infinite learning number of gains $p_{k}$ and $q_{k}$ satisfying (5) and (6). Denoting the iterative process of $m$-th order ILC scheme as $\mathcal{I}_{m}$, according to the definition of $Q$-factor,

$$
\begin{aligned}
& Q_{1}\left(\mathcal{I}_{m}, 0\right)=\lim _{i \rightarrow \infty} \sup \frac{\left|\Delta u_{i+1}\right|_{\lambda}}{\left|\Delta u_{i}\right|_{\lambda}} \\
& \leq \gamma_{1}+\gamma_{2} \lim _{i \rightarrow \infty} \sup \frac{\left|\Delta u_{i-1}\right|_{\lambda}}{\left|\Delta u_{i}\right|_{\lambda}}+\gamma_{3} \lim _{i \rightarrow \infty} \sup \frac{\left|\Delta u_{i-2}\right|_{\lambda}}{\left|\Delta u_{i}\right|_{\lambda}} \\
& +\cdots+\gamma_{m} \lim _{i \rightarrow \infty} \sup \frac{\left|\Delta u_{i-m+1}\right|_{\lambda}}{\left|\Delta u_{i}\right|_{\lambda}} \\
& \leq \gamma_{1}+\gamma_{2} \frac{1}{Q_{1}\left(\mathcal{I}_{m}, 0\right)}+\gamma_{3} \frac{1}{Q_{1}\left(\mathcal{I}_{m}, 0\right)^{2}} \\
& +\cdots+\gamma_{m} \frac{1}{Q_{1}\left(\mathcal{I}_{m}, 0\right)^{m-1}}
\end{aligned}
$$

where $\gamma_{k}=\left|p_{k}-q_{k} g_{u}\right|, \forall k=1, \ldots, m$. Define the following characteristic equation of $m$-th order ILC scheme

$$
Q_{1}^{m}\left(\mathcal{I}_{m}, 0\right)-\gamma_{1} Q_{1}^{m-1}\left(\mathcal{I}_{m}, 0\right)-\cdots-\gamma_{m}=0 .
$$

The learning convergence speed will be determined by one root of $(9)$.

Lemma 1. Assume that $\tau_{m} \geq \tau_{2} \cdots \geq \tau_{1}$ are roots of the following equation

$$
z^{m}-\gamma_{1} z^{m-1}-\ldots-\gamma_{m-1} z-\gamma_{m}=0
$$

subject to $\sum_{k=1}^{m} \gamma_{k}<1$ and $\gamma_{k}>0, k=1, \cdots, m$, then $0<\tau_{1}<1, \tau_{2}<0,\left|\tau_{m}\right|>\left|\tau_{l}\right|, \forall l=1, \cdots, m-1$.
Proof: See Appendix B.

According to Lemma 1, there is only one positive root $\tau_{m}$ of $(9)$, which has the largest absolute value among all $m$ roots. Thus $Q_{1}\left(\mathcal{I}_{m}, 0\right)=\tau_{m}$ which determines the slowest convergence speed. The value of $\tau_{m}$ depends on $\gamma_{k}, \forall k=1, \cdots, m$. The relationship between $\tau_{m}$ and $\gamma_{k}$ is given by the following lemma.

Lemma 2. Denote $\tau_{m}$ the only positive root of $z^{m}-$ $\gamma_{1} z^{m-1}-\ldots-\gamma_{m-1} z-\gamma_{m}=0$ and $\tilde{\tau}_{m}$ be the only positive root of $z^{m}-\tilde{\gamma}_{1} z^{m-1}-\ldots-\tilde{\gamma}_{m-1} z-\tilde{\gamma}_{m}=0$. If $\tilde{\gamma}_{i}>\gamma_{i}, \forall k=1, \cdots, m$, then $\tilde{\tau}_{m}>\tau_{m}$

\section{Proof: See Appendix C.}

Lemma 2 provides an important property: the smaller a $\gamma_{k}$, the smaller the root $\tau_{m}$. Thus in the ILC control design, parameters $p_{k}$ and $q_{k}$ should be chosen to minimize $\gamma_{k}, \forall k=1, \cdots, m$ in the presence of the interval uncertainty $g_{u} \in \mathcal{D}$. It can be formulated as the following min-max problem

$$
J_{m}=\min _{\left(p_{1}, \ldots, p_{m-1}\right) \in R^{m-1}} \min _{\left(q_{1}, \ldots, q_{m}\right) \in R^{m}} \max _{g_{u} \in \mathcal{D}} \tau_{m} .
$$

According to Proposition 2, $\min _{q_{k} \in R} \max _{g_{u} \in \mathcal{D}} \gamma_{k}=\left|p_{k}\right| \theta$. Since $q_{k}, k=1, \cdots, m$ are independent design parameters, we can substitute the optimal design for each $\gamma_{k}$ into (10) and the resulting equation is

$$
z^{m}-\left|p_{1}\right| \theta z^{m-1}-\ldots-\sum_{k=1}^{m-1}\left|p_{k}\right| \theta=0 .
$$

After fixing all $q_{k}$, the objective function $J_{m}$ is

$$
J_{m}=\min _{\left(p_{1}, \ldots, p_{m-1}\right) \in R^{m-1}} \bar{\tau}_{m}\left(p_{1}, \ldots, p_{m-1}\right)
$$

where $\bar{\tau}_{m}$ is the positive root of the equation (12).

Instead of determining all optimal $p_{k}$ which is difficult, we investigate the relationship between $\bar{\tau}_{m}$ and the quantity $\kappa_{m}=\sum_{k=1}^{m-1}\left|p_{k}\right| \theta+\left|1-\sum_{k=1}^{m-1} p_{k}\right| \theta$, which is given in the following lemma.

Lemma 3. $\bar{\tau}_{m}$, the only positive root of (12), will increase (decrease) as $\kappa_{m}$ increases (decreases).

Proof: See Appendix C.

According to Lemma 3, the optimization problem (13) is equivalent to

$$
\bar{J}_{m}=\min _{\left(p_{1}, \ldots, p_{m-1}\right) \in R^{m-1}} \sum_{k=1}^{m-1}\left|p_{k}\right|+\left|1-\sum_{k=1}^{m-1} p_{k}\right| \cdot(14
$$

The relationship of convergence speed for the family of linear-type ILC schemes can be represented by the following theorem 
Theorem 1. Consider the dynamic system (2) with the $m$-th order learning updating law (4), if (5) and (6)are satisfied, then $\bar{J}_{m} \leq \bar{J}_{m+1}, \forall m \geq 1$.

Proof: We need to prove that the convergence speed of the $m$-th order ILC scheme is faster that of the $(m+1)$-th order ILC scheme for any $m \geq 1$

The $(m+1)-t h$ order ILC scheme is expressed as

$$
u_{i+1}(t)=\sum_{k=1}^{m+1} \tilde{p}_{k} u_{i-k+1}+\sum_{k=1}^{m+1} \tilde{q}_{k} \Delta y_{i-k+1}
$$

where $\sum_{k=1}^{m+1} \tilde{p}_{k}=1$. As discussed in the $m$-th order ILC scheme, the convergence condition is $\sum_{k=1}^{m+1} \mid \tilde{p}_{k}-$ $\tilde{q}_{k} d \mid<1$. The characteristic equation of $(m+1)$ th ILC can be written as

$$
z_{m+1}^{m+1}-\tilde{\gamma}_{1} z_{m+1}^{m}-\ldots-\tilde{\gamma}_{m} z_{m+1}-\tilde{\gamma}_{m+1}=0 .
$$

According to Lemma 3,

$$
\begin{aligned}
& \bar{J}_{m+1} \min _{\left(\tilde{p}_{1}, \tilde{p}_{2}, \ldots, \tilde{p}_{m}\right) \in R^{m}} \sum_{k=1}^{m}\left|\tilde{p}_{k}\right| \theta+\left|1-\sum_{k=1}^{m} \tilde{p}_{k}\right| \\
& =\min _{\left(\tilde{p}_{1}, \tilde{p}_{2}, \ldots, \tilde{p}_{m}\right) \in R^{m}} \sum_{k=1}^{m}\left|\tilde{p}_{k}\right|+\left|1-\sum_{k=1}^{m-1} \tilde{p}_{k}-\tilde{p}_{m}\right| \\
& \geq \min _{\left(\tilde{p}_{1}, \tilde{p}_{2}, \ldots, \tilde{p}_{m}\right) \in R^{m}} \sum_{k=1}^{m-1}\left|\tilde{p}_{k}\right|+\left|\tilde{p}_{m}\right|+\left|1-\sum_{k=1}^{m-1} \tilde{p}_{k}\right|-\left|\tilde{p}_{m}\right| \\
& \geq \min _{\left(\tilde{p}_{1}, \tilde{p}_{2}, \ldots, \tilde{p}_{m-1}\right) \in R^{m-1}} \sum_{k=1}^{m-1}\left|\tilde{p}_{k}\right|+\left|1-\sum_{k=1}^{m-1} \tilde{p}_{k}\right|=\bar{J}_{m}
\end{aligned}
$$

The fastest convergence speed of the $m$-th order ILC scheme is faster than that of the $(m+1)$-th order ILC scheme.

Obviously, the fastest convergence speed achieves when $m=1$ in the family of linear-type ILC schemes, i.e., the first-order ILC scheme converges fastest in the presence of the interval uncertainties. If $m=1$, $Q_{1}\left(\mathcal{I}_{1}, 0\right)=\gamma_{1}=\left|1-q_{1} g_{u}\right|$. From Proposition 2, The optimal learning gain $q_{1}=\theta$ can be obtained directly. Consequently, the optimal learning gains for the family of linear-type ILC schemes, which ensures the fastest convergence speed under the interval uncertainties are $p_{1}^{*}=1, q_{1}^{*}=\theta, p_{k}^{*}=q_{k}^{*}=0, k=2, \cdots, m$.

\section{ILLUSTRATIVE EXAMPLES}

To clearly illustrate the effectiveness of robust optimal design, a linear time-invariant dynamic system is first considered with different $g_{u}$. A nonlinear dynamic system is also shown to demonstrate the superior performance of robust optimal design.

\subsection{Linear time-invariant dynamic system}

Consider a linear time-invariant dynamic system

$$
\begin{aligned}
& \dot{x}(t)=-3 x(t)-u(t) \quad x(0)=0.5 \\
& y(t)=x(t)+d u(t)
\end{aligned}
$$

where the known bound of $d$ is $\mathcal{D}=[0.5,2]$. The target trajectory is

$$
y_{d}(t)=\pi \sin \pi t+\frac{1}{2-t}, \quad t \in[0,1.5] .
$$

Note $y_{d}(0)=y(0)$. Since $d=g_{u} \neq 0$, it is appropriate to adopt the simplest first-order linear ILC scheme (4). By considering the worst case $d=\alpha_{2}=2$, a conservative learning gain $q_{1}=0.5$, which satisfies convergence condition $\left|1-q_{1} d\right|<1$, is chosen. It is easy to verify the convergence condition $\gamma_{1} \leq r=0.75$, $\forall d \in \mathcal{D}$. Let us verify the effectiveness of the robust optimal design using LTI dynamic system (17) with four different $d \in \mathcal{D}$.

According to the robust optimal design, the learning gain is $q_{1}=0.8$. From Fig. 1 we can see that the robust optimal design outperforms the conservative one when $d=0.5$ (solid line), 1 (dashed line) and 1.5 (dotted line), the only exception is $d=2$ (dash-dotted line) in which the conservative design achieves a "deadbeat" as $\gamma_{1}=0$. In the figure the thick lines correspond to robust optimal design and plain lines correspond to conservative design.

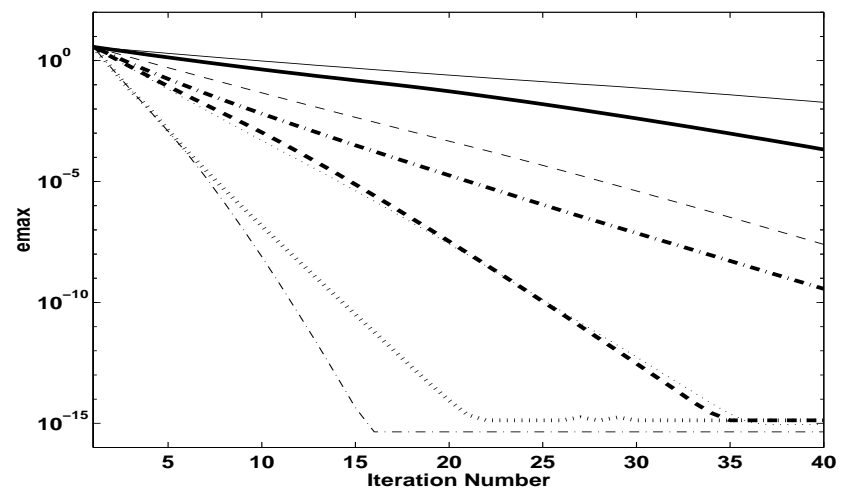

Fig. 1. Learning convergence with robust optimal design

Let us further compare the performance of secondorder ILC scheme and first-order ILC scheme. Since the robust optimal design of $\mathcal{I}_{2}$ leads to the first order ILC, we have to choose learning gains of $\mathcal{I}_{2}$ in a heuristic manner. According to (Bien and Huh, 1989; Chen et al., 1998), choosing $p_{1}$ and $p_{2}$ with opposite values may give faster response. Thus we set $p_{1}=1.2$, $p_{2}=-0.2, q_{1}=0.5$ and $q_{2}=0.4$. It is easy to verify that two convergence conditions (5) and (6) are satisfied. Simulating the four cases with $d=0.5$ (solid line), 1 (dashed line) and 1.5 (dotted line) and $d=2$ (dash-dotted line), the results are demonstrated in Fig. (2) with plain lines, and compared with the firstorder (thick lines). 


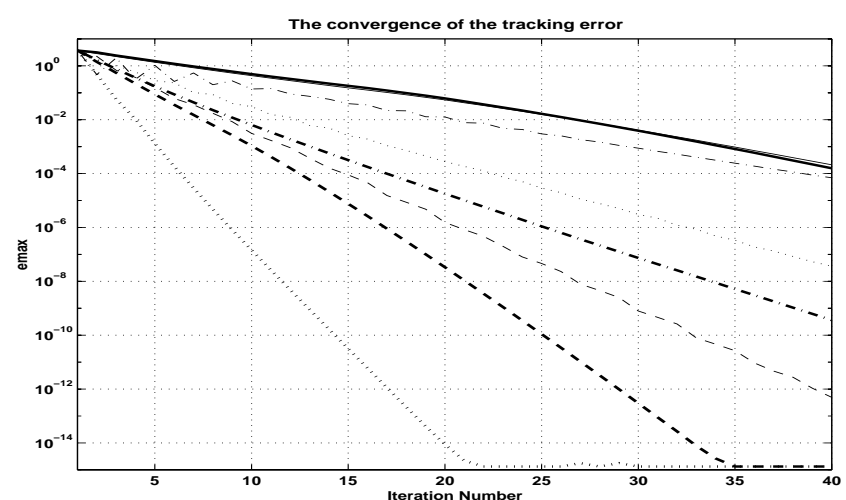

Fig. 2. Learning convergence profiles of 2nd-order ILC vs first-order ILC

From Fig. 2, we can see the performance of robust optimal design (first-order ILC scheme) outperforms the second order ILC scheme in all four cases.

\subsection{Nonlinear non-affine dynamic system}

Consider the example below, which slightly differs from (17) with a non-affine factor

$$
\begin{aligned}
& \dot{x}(t)=-3 x(t)+u(t) \quad x(0)=0.5 \\
& y(t)=x(t)+2 \operatorname{arctg}\left(\frac{1}{2} \operatorname{tg} \frac{u}{2}\right) .
\end{aligned}
$$

It is easy to verify that $g_{u} \in[0.5,2] \forall u \in \mathcal{R}$, the system is global Lipschitz continuous.

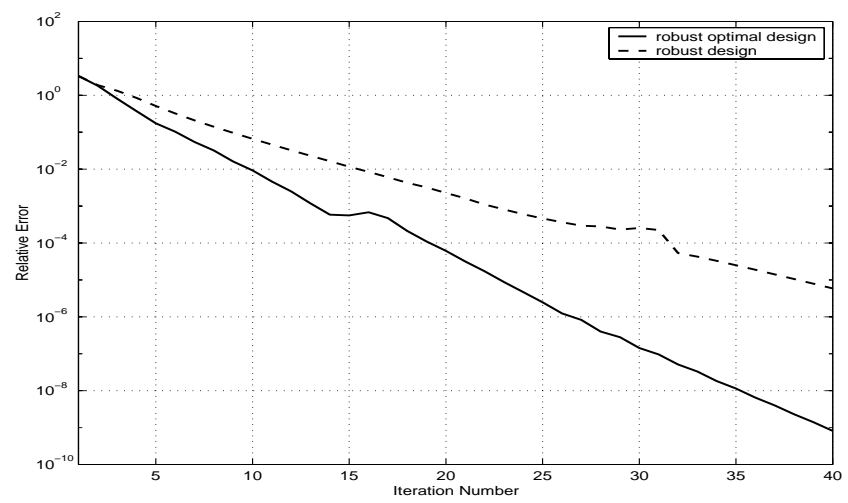

Fig. 3. Learning convergence with robust optimal design vs conservative design for a nonlinear nonaffine dynamic system

Similar to analysis of LTI example, conservative design $q_{1}=0.5$ and robust optimal design $q_{1}=0.8$ are compared. The results are shown in Fig. (3) with robust optimal design (solid line) and conservative design (dot line). The results shows the superiority of robust optimal design.

\section{CONCLUSION}

In this paper the family of linear-type ILC schemes, from the first order to $m$-th order, were designed with optimality under interval uncertainty. It was made clear that lower order ILC always outperforms higher order ILC schemes according to the newly introduced $Q$-factor. At the same time, by using the technique of robust optimization, the optimal gains for the family of linear-type ILC scheme are also given.

\section{APPENDIX A-PROOF OF PROPOSITION 2}

Define $z=p-q g_{u}$. Note that $q$ is an unrestricted variable, the feasible region of $J=\min _{q \in R} \max _{g_{u} \in \mathcal{D}}\left|p-q_{1} g_{u}\right|$ can be divided according to the range of $p$ and $q$. Here we show derivations for $p>0$.

When $p>0$, the objective function can be solved according to the feasible region of parameter $q$, which is divided into 3 intervals:

(1) $q \in\left(-\infty, \frac{p}{\alpha_{2}}\right] \rightarrow z \geq 0, \forall g_{u} \in \mathcal{D}$.

Let $J_{a}=\min _{q \in\left[0, \frac{p}{\alpha_{2}}\right]} \max _{g_{u} \in \mathcal{D}}\left|p-q g_{u}\right|$. Since $z \geq 0$,

$$
J_{a}=\min _{q \in\left(-\infty, \frac{p}{\alpha_{2}}\right]} \max _{g_{u} \in \mathcal{D}} p-q g_{u}=p\left(1-\frac{\alpha_{1}}{\alpha_{2}}\right) .
$$

$J_{a}$ reaches its minima when $q=\frac{p}{\alpha_{2}}$.

(2) $q \in\left[\frac{p}{\alpha_{1}}, \infty\right) \rightarrow z \leq 0, \forall g_{u} \in \mathcal{D}$.

$$
\begin{gathered}
\text { Let } J_{b}=\min _{q \in\left[\frac{p}{\alpha_{1}}, \infty\right)} \max _{g_{u} \in \mathcal{D}}\left|p-q g_{u}\right| . \text { Since } z \leq 0, \\
J_{b}=\min _{q \in\left[\frac{p}{\alpha_{1}}, \infty\right)} \max _{g_{u} \in \mathcal{D}} q g_{u}-p=p\left(\frac{\alpha_{2}}{\alpha_{1}}-1\right) .
\end{gathered}
$$

$J_{b}$ reaches its minimal when $q=\frac{p}{\alpha_{1}}$.

(3) $q \in\left[\frac{p}{\alpha_{2}}, \frac{p}{\alpha_{1}}\right]$

Given a point $q \in\left[\frac{p}{\alpha_{2}}, \frac{p}{\alpha_{1}}\right]$, the interval $\mathcal{D}$ can be divided into two subintervals $\mathcal{D}=\mathcal{D}_{L} \cup \mathcal{D}_{R}=$ $\left[\alpha_{1}, \frac{p}{q}\right] \bigcup\left[\frac{p}{q}, \alpha_{2}\right]$. Corresponding to the given $q$ we have

$$
\left\{\begin{array}{l}
z \geq 0, \forall g_{u} \in \mathcal{D}_{L} \\
z \leq 0, \forall g_{u} \in \mathcal{D}_{R} .
\end{array}\right.
$$

As discussed in (a) and (b),

$$
\begin{aligned}
& \max _{g_{u} \in \mathcal{D}_{L}}\left|p-q g_{u}\right|=p-q \alpha_{1} \\
& \max _{g_{u} \in \mathcal{D}_{R}}\left|p-q g_{u}\right|=q \alpha_{2}-p .
\end{aligned}
$$

The objective function is

$$
\begin{aligned}
J_{c} & =\min _{q \in\left[\frac{p}{\alpha_{2}}, \frac{p}{\alpha_{1}}\right]} \max _{g_{u} \in \mathcal{D}}\left|p-q g_{u}\right| \\
& =\min _{q \in\left[\frac{p}{\alpha_{2}}, \frac{p}{\alpha_{1}}\right]}\left\{p-q \alpha_{1}, q \alpha_{2}-p\right\} \\
& =\frac{p\left(\alpha_{2}-\alpha_{1}\right)}{\alpha_{1}+\alpha_{2}}
\end{aligned}
$$

when $q=\frac{2 p}{\alpha_{1}+\alpha_{2}}$. 
Since $J_{c} \leq J_{a} \leq J_{b}, J$ reaches its minimum $\frac{p\left(\alpha_{2}-\alpha_{1}\right)}{\alpha_{1}+\alpha_{2}}$ when $q=\frac{2 p}{\alpha_{1}+\alpha_{2}}$.

From the discussion above, $J=p \frac{\alpha_{2}-\alpha_{1}}{\alpha_{2}+\alpha_{1}}$ and $q=$ $\frac{2 p}{\alpha_{2}+\alpha_{1}}$ when $p>0$.

When $p \leq 0$, analogously by solving the min-max problem using the same technique we can reach $J=$ $|p| \frac{\alpha_{2}-\alpha_{1}}{\alpha_{2}+\alpha_{1}}$ and $q=\frac{2 p}{\alpha_{2}+\alpha_{1}}$ when $p \leq 0$.

Accordingly the optimal value of $J$ equals to $|p| \frac{\alpha_{2}-\alpha_{1}}{\alpha_{2}+\alpha_{1}}$ when $q=\frac{2 p}{\alpha_{2}+\alpha_{1}}$.

\section{APPENDIX B-PROOF OF LEMMA 1}

Assume that $\tau_{m} \geq \tau_{m-1} \geq \cdots \geq \tau_{1}$ are roots of equation (10). From Descartes' rule of signs (Albert, 1943, ), there is only one positive root of $(10)$, which implies $\tau_{m}>0$ and $\tau_{m-1}<0$.

Let $P_{m}(z)=z^{m}-\gamma_{1} z^{m-1}-\ldots-\gamma_{m-1} z-\gamma_{m}$. Since $P_{m}(0)=-\gamma_{m}<0$ and $P_{m}(1)=1-\sum_{k=1}^{m} \gamma_{k}>0$, the only positive root $\tau_{m}$ is in the open interval $(0,1)$. Therefore, there is only one root of equation (10) in the interval $(0,1)$. Note that $\sum_{l=1}^{m} \tau_{m}=\gamma_{1}$, since $\tau_{l}<0, \forall l=m-1, \cdots, 1$, we have

$$
\begin{aligned}
\sum_{l=1}^{m} \tau_{l}=\gamma_{1} \geq 0 & \Rightarrow\left|\tau_{m}\right|-\left|\tau_{m-1}\right|-\cdots-\left|\tau_{1}\right|=\gamma_{1} \\
& \Rightarrow\left|\tau_{m}\right|>\left|\tau_{l}\right|, \quad \forall l=m-1, \cdots, 1
\end{aligned}
$$

Clearly, $\tau_{m}$ is the only positive root of equation (10) and has the largest absolute value.

\section{APPENDIX C-PROOF OF LEMMA 2}

Let $P_{m}(z)=z^{m}-\gamma_{1} z^{m-1}-\cdots-\gamma_{m-1} z-\gamma_{m}$ and $\tilde{P}_{m}(z)=z^{m}-\tilde{\gamma}_{1} z^{m-1}-\cdots-\tilde{\gamma}_{m-1} z-\tilde{\gamma}_{m}$. Denote $\tau_{m}$ and $\tilde{\tau}_{m}$ to be positive roots of $P_{m}(z)=0$ and $\tilde{P}_{m}(z)=0$ respectively. Since $0<\gamma_{i}<\tilde{\gamma}_{i}<1$, $\tilde{P}_{m}(0)<0$. ¿From Lemma $1,0<\tau_{m}<1$ and $0<\tilde{\tau}_{m}<1$. Clearly we have

$$
\begin{aligned}
\tilde{P}_{m}\left(\tau_{m}\right)= & \left(\tau_{m}^{m}-\gamma_{1} \tau_{m}^{m-1}-\cdots-\gamma_{m-1} \tau_{m}-\gamma_{m}\right) \\
& +\left(\gamma_{1}-\tilde{\gamma}_{1}\right) \tau_{m}^{m-1}+\left(\gamma_{2}-\tilde{\gamma}_{2}\right) \tau_{m}^{m-2}+\cdots \\
& +\left(\gamma_{m}-\tilde{\gamma}_{m}\right) \\
= & \left(\gamma_{1}-\tilde{\gamma}_{1}\right) \tau_{m}^{m-1}+\left(\gamma_{2}-\tilde{\gamma}_{2}\right) \tau_{m}^{m-2}+\cdots \\
& +\left(\gamma_{m}-\tilde{\gamma}_{m}\right)<0
\end{aligned}
$$

therefore $\tilde{\tau}_{m} \in\left(\tau_{m}, 1\right)$, i.e., $\tilde{\tau}_{m}>\tau_{m}$.

\section{APPENDIX D-PROOF OF LEMMA 3}

Define $\kappa_{m}=\sum_{k=1}^{m}\left|p_{k}\right| \theta$ and $\tilde{\kappa}_{m}=\sum_{k=1}^{m}\left|\tilde{p}_{k}\right| \theta$, and assume $\kappa_{m}<\tilde{\kappa}_{m}$. Let $\tau_{m}$ and $\tilde{\tau}_{m}$ be the only positive roots of equation $P_{m}(z)=z^{m}-\left|p_{1}\right| \theta z^{m-1}-\cdots-\left|p_{m-1}\right| \theta z-$ $\left|p_{m}\right| \theta=0$ and $\tilde{P}_{m}(z)=z^{m}-\left|\tilde{p}_{1}\right| \theta z^{m-1}-\cdots-$ $\left|\tilde{p}_{m-1}\right| \theta z-\left|\tilde{p}_{m}\right| \theta=0$ respectively. Since $\kappa_{m}<\tilde{\kappa}_{m}$, it can be deduced that $\left|p_{m}\right|-\left|\tilde{p}_{m}\right|<\left|\tilde{p}_{1}\right|-\left|p_{1}\right|+\ldots+$ $\left|\tilde{p}_{m-1}\right|-\left|p_{m-1}\right|$. From the fact that $0<\tau_{m}<1$, we can obtain

$$
\begin{aligned}
& \tilde{P}_{m}\left(\tau_{m}\right) \\
& =\tau_{m}^{m}-\left|p_{1}\right| \theta \tau_{m}^{m-1}-\cdots-\left|p_{m-1}\right| \theta \tau_{m}-\left|p_{m}\right| \theta \\
& +\left(\left|p_{1}\right|-\left|\tilde{p}_{1}\right|\right) \theta \tau_{m}^{m-1}+\cdots+\left(\left|p_{m-1}\right|-\left|\tilde{p}_{m-1}\right|\right) \theta \tau_{m} \\
& +\left(\left|p_{m}\right|-\left|\tilde{p}_{m}\right|\right) \theta \\
& =\theta\left[\left(\left|p_{1}\right|-\left|\tilde{p}_{1}\right|\right) \tau_{m}^{m-1}+\cdots+\left(\left|p_{m-1}\right|-\left|\tilde{p}_{m-1}\right|\right) \tau_{m}\right. \\
& \left.+\left(\left|p_{m}\right|-\left|\tilde{p}_{m}\right|\right)\right] \\
& <\theta\left\{\left[\left(\left|p_{1}\right|-\left|\tilde{p}_{1}\right|\right) \tau_{m}^{m-1}+\cdots+\left(\left|p_{m-1}\right|-\left|\tilde{p}_{m-1}\right|\right) \tau_{m}\right]\right. \\
& \left.-\left[\left(\left|p_{1}\right|-\left|\tilde{p}_{1}\right|\right)+\cdots+\left(\left|p_{m-1}\right|-\left|\tilde{p}_{m-1}\right|\right)\right]\right\} \\
& <0
\end{aligned}
$$

Since $\tilde{P}_{m}(1)>0, \tilde{P}_{m}\left(\tau_{m}\right)<0$, it can be derived that $\tau_{m}<\tilde{\tau}_{m}<1$, i.e., $\kappa_{m}<\tilde{\kappa}_{m} \Rightarrow \tau_{m}<\tilde{\tau}_{m}$.

\section{REFERENCES}

Albert, A. A. (1943). An inductive proof of Descartes' rule of signs. American Mathematical Monthly 50(3), 178-180.

Arimoto, S. (1985). Mathematical theory of learning with applications to robot control. In: Adaptive and Learning Systems: Theory and Applications (K.S. Narendra, Ed.). pp. 379-388. Yale University. Yale University, New Haven, Connecticut, USA.

Bien, Z. and K. M. Huh. (1989). High-order iterative learning control algorithm. IEE Proc. Pt.-D, 136(3) 105-112.

Chen, Y. Q., Z. M. Gong and C. Y. Wen (1998). Analysis of a high-order iterative learning control algorithm for uncertain nonlinear systems with state delays. Automatica 34(3), 345-353.

Kuc, Y.Y., S.J. Lee and K.H. Nam (1992). An iterative learning control theory for a class of nonlinear dynamic systems. Automatica 28(10), 1215-1221.

Longman, R. W. and Chun-Ping Lo (1997). Generalized holds, ropple attenuation, and tracking additional outputs in learning control. Journal of Guidance, Control, and Dynamics 20(6), 12071214.

Moore, K. L. (1998). Iterative learning control - an expository overview. Applied \& Computational Controls, Signal Processing, and Circuits , 1-42.

Xu, Jian-Xin (1997). Analysis of iterative learning control for a class of nonlinear discrete-time systems. Automatica 33(10), 1905-1907. 